\title{
Text-based Requirements Preprocessing using Nature Language Processing Techniques
}

\author{
Huafeng Chen, Keqing He, Peng Liang, Rong Li \\ State Key Lab of Software Engineering \\ Wuhan University \\ Wuhan, China \\ canada186771@yahoo.com.cn
}

\begin{abstract}
In a distributed environment, non-technical stakeholders are required to write down requirement statements by themselves. Nature language is the first choice for them. In order to alleviate the burden of reading free-text requirement documents by requirements engineers, we extract goals and relevant stakeholders from requirement statements automatically by a computer-assisted way. In this paper, requirements are divided into system level requirements and instance level requirements. Methods are proposed to solve two types of requirements by analyzing the characteristics of requirement expressions, and combining techniques of nature language processing with semantic web. Semantic-enhanced segment and domain sentence pattern are two novel techniques utilized in our methods. Our approach accelerates goal extraction from text-based requirements and alleviates the burden of requirements engineers significantly.
\end{abstract}

Keywords-requirements preprocessing; nature language processing; domain ontology; non-technical stakeholder; Wiki

\section{INTRODUCTION}

In traditional requirements engineering ( $R E)$ process, requirements engineers talk face-to-face to non-technical stakeholders, elicit their requirements and write them down either by nature language (NL) or by structured nature language (SNL) [1].

SNL usually has some benefits comparing to NL. It is a good communication mediator for both requirements engineers and non-technical stakeholders, because it is easily understandable by both. Also, it is easier to be processed by computer than NL. However, SNL has some drawbacks comparing to $\mathrm{NL}$.

(a) Users have to learn the syntax of SNL;

(b) There is no standard syntax of SNL which makes it impossible "to learn once to use anywhere".

Requirements engineers are usually familiar with techniques on RE and well educated, it won't be too difficult for them to learn SNL. Considering its benefits, learning a kind of SNL is still attractive. The problem of no standard SNL also seems not so important, because they can use one kind of SNL they are familiar with to document the requirements of different projects.

However, sometimes requirements engineers and nontechnical stakeholders are distributed geographically and located in different time zone, so talking face-to-face seems impossible. At this time, Wiki is adopted as a suitable platform to elicit distributed requirements through collaboration [2] [3]. Some problems also come with this novel method because of the involvement of non-technical stakeholders in documenting requirements.

In a Wiki, non-technical stakeholders are required to write down their requirements online by themselves, but they usually have little knowledge on RE, and cannot document requirements with professional tools. SNL is also not an ideal choice, due to two drawbacks mentioned before, by our research experiences. Nature language becomes the first choice and also the only choice for them to express their requirements.

Engineers read these requirements and analyze them asynchronously. Conflicts, inconsistency checking are tasks of requirements analysis in the following phase. It is costly and inefficient to read long stories. Computer assisted way to discover stakeholders and their requirement goals is advocated in this situation, which builds a solid foundation for next steps of analysis.

Extracting key information from free-text requires techniques of nature language processing (NLP). This paper focuses on how to preprocess early requirements and create a semi-formal model for further analysis. Our method is based on the characteristics of requirements expressions in NL. Requirements of a project are always within the scope of a domain, so domain ontology, which can filter improper requirements, is utilized in our method.

The rest of the paper is organized as follows. Section 2 introduces some background knowledge related to our research work. We will discuss how to preprocess early requirements and extract goals with NLP techniques in section 3. An evaluation and further discussion will be given in section 4. Section 5 illustrates some related works and section 6 concludes our paper with next steps.

\section{BACKGROUND KNOWLEDGE}

Some background knowledge on NLP and domain ontology is illustrated in this section, which can be used in our method.

\section{A. Corpus and Domain Ontologies}

Corpus plays a significant role in modern NLP, which is widely used in segment, morphological transformation and so on. Corpus can be divided into two categories according to its purpose. One is general corpus including words which are not limited to a domain; the other is domain specific, which is similar with domain ontology. 
Ontology is a formal specification of a shared conceptualization [4]. Domain ontology usually defines concepts and their relationships in a certain domain

The main differences between corpus and ontology are that ontology includes more complex relationships of concepts and usually has the ability of reasoning, whereas corpus includes word, part-of-speech, and simple relationships between words, such as, synonym and antonym.

In our method, we choose domain ontology described in OWL [5] as the knowledge base for NLP. The domain ontology is illustrated in Figure 1.

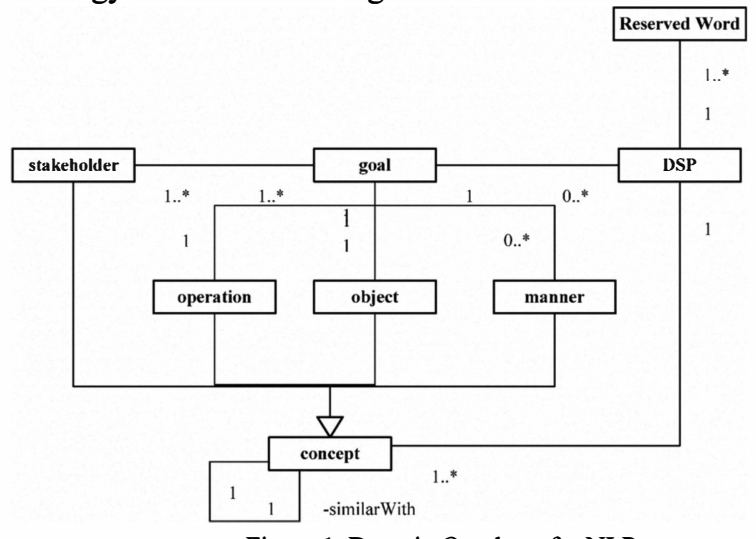

Figure 1. Domain Ontology for NLP

This ontology, which describes stakeholders and their goals, is part of a large ontology used in our research project [6] with a little extension. One stakeholder can have one or more goals and one goal can belong to one or more stakeholders. By analyzing some real life cases, we discover that it is possible to describe a goal with a verb phrase and a noun phrase, which are named operation and object, respectively, in our ontology. For example, we can name a goal "book air ticket" with an operation "book" and an object "air ticket". Sometimes, manner is also taken into account, e.g. "search parking area by place". DSP is acronym of domain sentence pattern, including at least one reserved word and concept. Details on DSP will be looked into further in section 3. Stakeholder, operation, object and manner are all subclasses of concept. Morphological forms of words in the concept are attached to it. In ontology described in OWL, there are some predefined relationships between concepts, such as "subclass", "the same as". We do not explicitly describe those predefined relationships in our ontology. But we add a relationship named "similarWith", to introduce synonym into ontology, which facilitates NLP.

\section{B. Segment Algorithm}

Segment is a key issue in language processing, such as Chinese. There are many classical algorithms for this problem. One category is called maximum matching (MM), which can be divided into "forward maximum matching (FMM)", "backward maximum matching (BMM)" and "bidirectional maximum matching (BiMM)".

Segment algorithms always produce a list of words. We propose a semantics enhanced FMM segment algorithm (seFMM), which produces a list of phrases, since the concepts in the ontology can be a phrase instead of a word.

\section{REQUIREMENTS PREPROCESSING}

In this section, we discuss how to preprocess early requirements with NLP techniques. Because we adopt the idea of goal-oriented requirements analysis method [7] [8] to refine requirements and check conflicts and inconsistency of requirements in our research group [6], we must extract goals from requirements in NL.

Untrained non-technical stakeholders have their own habits to express requirements, especially in an unsupervised environment, such as wiki. The differences of system level requirements (SLRs) and instance level requirements (ILRs) always confuse them.

Table 1 lists some requirements. It is not difficult to find that SLRs usually specify what stakeholders can do with the help of the would-be software or what the would-be software can provide to stakeholders, whereas ILRs usually indicate a running instance of the would-be software. For example, in Table 1, requirement (d) is a running instance of the wouldbe software having a function "search a parking area by place" with the input "Wuhan University".

In order to extract goals from two different types of requirements, we must analyze the expression characteristics of each type. SLRs usually have domain unspecific sentence patterns. For example, requirement (a) and requirement (b) belong to different domains, but they have the same sentence pattern. It is obvious that we can extract a goal "book train ticket" from requirement (a) and a goal "provide schedule" from requirement (b).

The situation is different in ILRs. Mix information at both system level and instance level spoils the analysis. For example, in requirement (d), "search a parking area" is system level information (SLI) and "near Wuhan University" is instance level information (ILI). Expressions of ILI differ from one piece of requirement to another, so domain specific sentence patterns should be introduced. How to summarize the requirement (d) in a goal is the problem we will address.

TABLE I. TWO TYPES OF REQUIREMENTS

\begin{tabular}{|c|l|c|c|}
\hline $\begin{array}{c}\text { Number of } \\
\text { Requirements }\end{array}$ & Requirements in NL & Type & Domain \\
\hline (a) & $\begin{array}{l}\text { Customers can book } \\
\text { train tickets. }\end{array}$ & SLR & $\begin{array}{c}\text { Traffic } \\
\text { System }\end{array}$ \\
\hline (b) & $\begin{array}{l}\text { The system should } \\
\text { provide right schedule. }\end{array}$ & SLR & $\begin{array}{l}\text { School } \\
\text { Schedule } \\
\text { System }\end{array}$ \\
\hline (c) & $\begin{array}{l}\text { Customers can search } \\
\text { and reserve hotel. }\end{array}$ & SLR & $\begin{array}{l}\text { Traffic } \\
\text { System }\end{array}$ \\
\hline (d) & $\begin{array}{l}\text { I'd like to search a } \\
\text { parking area near } \\
\text { Wuhan University. }\end{array}$ & ILR & $\begin{array}{c}\text { Traffic } \\
\text { System }\end{array}$ \\
\hline (e) & $\begin{array}{l}\text { Students would like to } \\
\text { check when to take } \\
\text { chemistry course. }\end{array}$ & ILR & $\begin{array}{l}\text { School } \\
\text { Schedule } \\
\text { System }\end{array}$ \\
\hline
\end{tabular}

We will discuss how to process SLRs in subsection B and how to process ILRs in subsection C. An overview of requirements preprocessing is given in subsection D. But first of all semantics enhanced FMM segment algorithm should be introduced. 


\section{A. Semantics Enhanced FMM Segment Algorithm}

We have discovered that concepts in ontology may be more than one word, and we mustn't divide one concept into two parts. The idea of FMM is adopted to solve this problem.

It is very common for non-technical stakeholders to express requirements with synonyms, so semantics should be introduced. We do it by defining a relationship named "similarWith" in the ontology.

Related algorithms are as follows.

Algorithm 3-1: Synonymous Concepts Generation

For every concept $c_{i}$ in the ontology;

For every word $w_{j}$ in $c_{i}$, get its synonyms to form a set $s_{j}$, where $1<=j<=\left|c_{i}\right|,\left|c_{i}\right|$ indicates the number of words $c_{i}$ contains;

For $\mathrm{j}$ from 1 to $\left|\mathrm{c}_{\mathrm{i}}\right|$

For every word in $s_{j}$ replace $w_{j}$, then generate a new concept $c_{\text {new }}$ which is similar to $c_{i}$;

Add $c_{\text {new }}$ to the ontology and generate the relationship "similarWith" between $\mathrm{c}_{\text {new }}$ and $\mathrm{c}_{\mathrm{i}}$;

Algorithm 3-1 diffuses the "similarWith" relationship of words to concepts, and the results are stored into ontology for reuse. Thus, we improve the performance of seFMM in Algorithm 3-2.

\section{Algorithm 3-2: seFMM}

Assume a sentence has $n$ words, i.e. $\mathrm{w}_{1}, \mathrm{w}_{2}, \mathrm{w}_{\mathrm{i}}, \ldots$, $\mathrm{w}_{\mathrm{n}}$, and an index $\mathrm{i}$;

Let $i=1$, resultList $=\emptyset$;

While $\mathrm{i}<=\mathrm{n}$

Select all the concepts whose first word is the same with $\mathrm{w}_{\mathrm{i}}$ to form a set $\mathrm{C}$;

For every concept $c_{k}$ in $\mathrm{C}$ from the longest one to the shortest one

If $c_{k}=$ concat $\left(w_{i}, w_{i+1}, w_{i+2}, \ldots, w_{j}\right)$, where concat () is a function to concatenate all its parameters, then $\mathrm{i}=\mathrm{j}$, add $\mathrm{c}_{\mathrm{k}}$ to resultList; Else add $\mathrm{w}_{\mathrm{i}}$ to resultList; $\mathrm{i}=\mathrm{i}+1$;

We apply Algorithm 3-2 on the ontology which has been preprocessed by Algorithm 3-1 to realize semantic enhancement.

\section{B. System Level Requirements Preprocessing}

The target of SLRs preprocessing is to discover goals and relevant stakeholders automatically. However, we are not intend to discover all the goals and the stakeholders correctly. Our purpose is to alleviate the burden of requirements engineers in a computer assisted way.

From Table 1, we can easily find two phenomena.

(a) One stakeholder usually takes responsible for the SLRs within one short sentence separated by a semicolon or a period.

(b) There can be more than one goal in one short sentence (see requirement (c) in table 1).
To the phenomena, we segment long sentences into short ones separated by a semicolon or a period.

Before applying Algorithm 3-3, we should segment a sentence into a list of concept instances with seFMM, and it is easy to annotate those instances with their concepts defined in the domain ontology, such as operation, object.

Having done the preparing works, Algorithm 3-3 can be applied to a list of annotated concepts.

Algorithm 3-3: Goals and Stakeholders Extraction

Input: a list of annotated concepts. For example, "Customer/stakeholder", "book/operation", "train ticket/object".

Output: goals with their stakeholders. For example, "book train ticket/goal", "customer/relevant stakeholder to 'book train ticket"'.

1. Get all the stakeholders with their position in the list to form a set St;

2. Get all the operations with their position in the list to form a set $\mathrm{Op}$;

3. Get all the objects with their position in the list to form a set $\mathrm{Obj}$;

4. For every operation $o p_{i}$ in $\mathrm{Op}$

For every object $\mathrm{obj}_{\mathrm{j}}$ in $\mathrm{Obj}$

If $\operatorname{pos}_{i}<\operatorname{pos}_{j}$, then generate a coarse goal with the name of concat $\left(o p_{i}, o b j_{j}\right)$, add the coarse goal with pos $_{i}$ into set CG where pos $s_{i}$ is the position of $\mathrm{op}_{\mathrm{i}}$;

If the coarse goal exists in the domain ontology, then it can be added into the set of fine goal (FG) with pos ${ }_{i}$;

5. For every stakeholder $\mathrm{st}_{\mathrm{i}}$ in $\mathrm{St}$

For every fine goal $\mathrm{fg}_{\mathrm{j}}$ in FG

If $\operatorname{pos}_{\mathrm{i}}<\operatorname{pos}_{\mathrm{j}}$, then generate a connection $<\mathrm{st}_{\mathrm{i}}, \mathrm{fg}_{\mathrm{j}}>$ indicating $\mathrm{fg}_{\mathrm{j}}$ has a relevant stakeholder $\mathrm{st}_{\mathrm{i}}$;

Algorithm 3-3 adopts the idea that the scopes of operation and stakeholder are from where they appear to the end of the short sentence. This is based on the assumption that active-voice sentences are used to describe requirements. We can improve this algorithm by judging the short sentence whether it is active-voice or passive-voice first, and then taking corresponding strategies to decide the scopes of operation and stakeholder.

\section{Domain Sentence Patterns and Instance Level Requirements Preprocessing}

As mentioned before, ILRs include ILI, as well as SLI, so SLRs can be regarded as a kind of special ILRs, and ILRs have additional information, which can be used to refine the goal. ILRs preprocessing can be divided into two phases. One is the same as SLRs preprocessing, and the other is how to refine the goal according to ILI.

Domain sentence pattern (DSP) is composed of concepts and reserved words (Figure 1) and is related to a certain goal. For example, the goal "search parking area by place" is the 
sub-goal of "search parking area". "Near place" is a DSP related to "search parking area by place", with "near" as a reserved word and "place" as a concept. The difference between reserved word and concept is that the former has no instances, while the latter has ones. The concept "place" may have instances, such as "Wuhan University", "Beijing". All those instances are replaced by the concept when the sentences are segmented and annotated. The sentence "I'd like to search a parking area near Wuhan University." becomes "search/op parking area/obj near/reserved word Wuhan University/place". Operation and object are extracted to form the goal "search parking area" with algorithm 3-3. "Near place" is used to refine the goal into "search parking area by place" in the context of "search parking area" which is the super goal of the one we get. ILI.

Algorithm 3-4 illustrates how to refine goals according to

Algorithm 3-4: Refining Goals from Instance Level Information

Precondition: Algorithm 3-2 and Algorithm 3-3 have been applied; a goal $g_{\text {can }}$ waiting for being refined and DSP of the requirement have been extracted in the step of segment and annotation.

For every $\mathrm{dsp}_{\mathrm{i}}$ in DSPs of $\mathrm{g}_{\mathrm{can}}$

If $\mathrm{dsp}_{\mathrm{i}}$ equals DSP from requirement, then

Refine the goal $g_{\text {can; }}$

Since there are so many ways to express the same idea, to illustrate all DSPs for a goal seems impossible at first, but they can be accumulated. Thus, the domain ontology can be more and more complete.

\section{Requirements Preprocessing}

In this subsection, we will give a whole procedure of requirements preprocessing using all the techniques mentioned above.

First, we must decide whether the piece of requirement is instance level or system level. Fortunately, the latter is a special case of the former, so we only need to take all the requirements as instance level ones. Preparing work, such as applying Algorithm 3-1 to domain ontology is required.

Second, segmentation and annotation are adopted to get a list of annotated phrases. Algorithm 3-2 is used at this step.

Third, Algorithm 3-3 is used to extract goals and relevant stakeholders.

Fourth, combine the goal gotten from the third step, DSP and instance information if there's any, to refine the goal (algorithm 3-4).

Figure 2 gives us an overview of the whole procedure.

Besides, this work can be integrated into a large research project of our group by exporting the results into another tool [9]. That tool can make full use of predefined domain assets to assist requirements analysis.

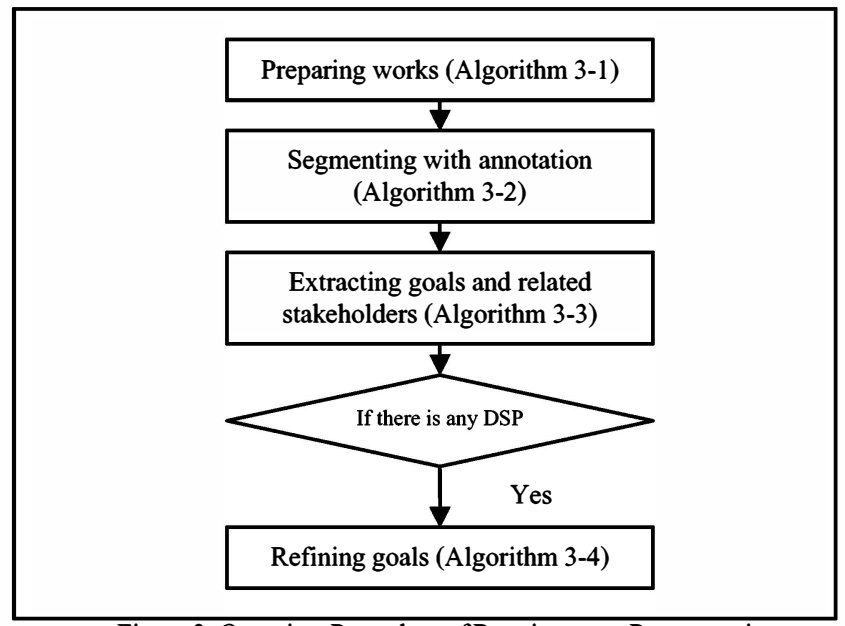

Figure 2. Overview Procedure of Requirements Preprocessing

\section{EVALUATION AND DiscUSSION}

In this section, we evaluate our method for SLRs by 6 cases from the RE course projects running in 2009 at University of Groningen in the Netherlands [10]. Students are separated into several groups, and each group was responsible for one case. We have 6 cases in all, which cover 6 different areas, including high school schedule system, bike rental system, tennis match registration system, online lectures system, website for student organization and a survey software to monitor and analyze customer satisfaction concerning treatments and procedures. Each case has goals ranging from 5 to 17 .

Figure 3 illustrates the result of goal extraction from 6 cases above.

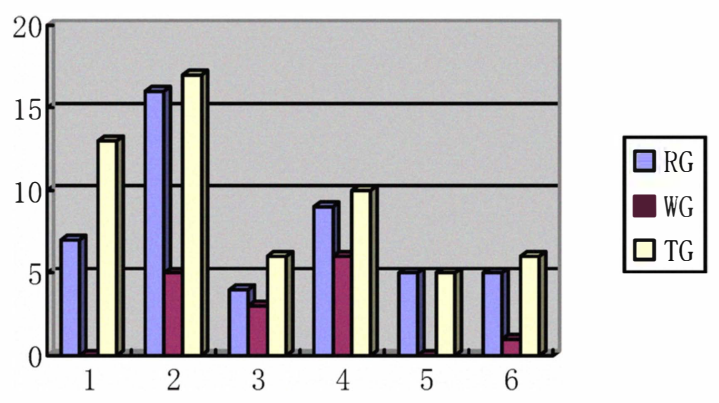

Figure 3. Results of Goal Extraction

The $\mathrm{x}$-coordinate indicates the case number, and the $\mathrm{y}$ coordinate is the number of goals in a case. RG means goals extracted correctly, WG means goals extracted incorrectly, and TG means the total number of goals extracted by human analysis.

Table 2 illustrates precision, recall and F-measure of experiment results. After analyzing the result, we discover that long sentence or proposing several goals together in one sentence will decrease the precision. Two factors decrease the recall.

(a) Some goals cannot be expressed simply in the form of operation and object. 
(b) Negative sentences hinder goals discovery.

TABLE II. STATISTICS OF RESULTS

\begin{tabular}{|c|c|c|c|}
\hline Case No. & Precision & Recall & F-measure \\
\hline 1 & 1.00 & 0.54 & 0.70 \\
\hline 2 & 0.76 & 0.94 & 0.84 \\
\hline 3 & 0.57 & 0.67 & 0.62 \\
\hline 4 & 0.60 & 0.90 & 0.72 \\
\hline 5 & 1.00 & 1.00 & 1.00 \\
\hline 6 & 0.83 & 0.83 & 0.83 \\
\hline Average & 0.79 & 0.81 & \\
\hline
\end{tabular}

Above all, this method is suitable for upper level goals extraction, which is simple to express and without many details.

\section{RELATED WORKS}

Liu proposes a kind of structured nature language named SORL to elicit end-users' requirements [1], which include many sentence patterns. In order to generate machine readable requirements, requirement unit $(\mathrm{RU}=<$ Subject $(\mathrm{S})$, Predicate $(\mathrm{P})$, Object $(\mathrm{O})$, Adverbial $(\mathrm{A})>$ ) is defined. A kind of sentence pattern "behavior" can be transformed into RU. Basing on RU, later analysis works can be done by computer. The paper doesn't mention how to transform sentence patterns to RU and the structured nature language still seems too difficult for end-users.

Semantic wiki, as a booming technique is used into distributed requirements elicitation. Some RE-specific semantic wikis have been developed [11][12]. A survey on them shows that those tool mainly focus on the activities of $\mathrm{RE}$, and seldom pay any attention to make the tool smarter by preprocessing requirements.

\section{CONCLUSIONS AND FUTURE WORKS}

In a distributed environment, non-technical stakeholders need to write down requirement statements by themselves. Due to the knowledge background, nature language is the first choice for them. To alleviate the burden of reading requirement statements by requirements engineers, NLP techniques are adopted to extract requirement goals and relevant stakeholders, which can be the inputs for the next step in requirements analysis. The adoption of domain ontology rather than a common corpus as a knowledge base for NLP bases on the fact that requirements of a project is always within the scope of a certain domain. Thus, concepts which are not in the domain will be omitted and less effort is needed to extract required information.

We have noticed that our method is compatible with goal-oriented requirements analysis, but cannot extract business rules, which is expected to be transformed into first order logic or even temporal logic. With further analysis, we argue that the sentence patterns describing business rules are something similar to DSP, but more complex, for example, how to decide the scope of quantifiers.

In the end, we emphasize that our method is to be used as a computer-assisted way, which should not be expected to extract all the goals precisely. Our purpose is to alleviate the burden of requirement engineers by providing information extracted in a tolerable degree.

\section{ACKNOWLEDGMENT}

The research project is supported by the National Basic Research Program of China (973) under Grant No. 2007CB310801, the National Natural Science Foundation of China under Grant No. 60970017, 60903034, the Eleventh Five-Year Plan for National Key Technology R\&D Program under Grant No. 2006BAK04A20-7.

\section{REFERENCES}

[1] W Liu, K He. "Heavyweight Semantic Inducement for Requirement Elicitation and Analysis". Proceedings of the Third International Conference on Semantics, Knowledge and Grid, SKG, 2007, pp.206211.

[2] B. Decker, E. Ras, J. Rech, P. Jaubert, and M. Rieth. "Wiki-Based Stakeholder Participation in Requirements Engineering" IEEE Software, 24(2):28-35, 2007.

[3] C.Silveira,J.Faria,A.Aguiar,andR.Vidal.Wiki Based Requirements Documentation of Generic Software Products. In Proceedings of the 10th Australian Workshopon Requirements Engineering (AWRE),pages 42-51, 2005.

[4] W. N. Borst. Construction of Engineering Ontologies for Knowledge Sharing and Reuse. PhD thesis, University of Twente, Enschede, 1997.

[5] http://www.w3.org/TR/owl-features/

[6] J Wang, K He, P Gong, et al. "RGPS: A Unified Requirements MetaModeling Frame for Networked Software". Proceedings of Third International Workshop on Advances and Applications of Problem Frames(IWAAPF'08) at 30th International Conference on Software Engineering(ICSE'08), Leipzig, Germany, May 2008.

[7] A. V. Lamsweerde, "Goal-oriented Requirements Engineering: a Guided Tour", In Proc. of the 5th IEEE International Symposium on Requirements Engineering, Toronto, Canada, 2001, pp. 249-263.

[8] A. Dardenne, A. V. Lamsweerde, and S. V. Fickas, "Goal-Directed Requirements Acquisition", Science of Computer Programming, Vol. 20, pp. 3-50, 1993.

[9] H. Chen, K. He, and B. Hu, "Domain Model Based Approach for Service-oriented Requirements Elicitation and Analysis", In Proceedings of ETP/IITA World Congress in Applied Computing, Computer Science, and Computer Engineering (ACC 2009), 2009.

[10] http://www.cs.rug.nl/ liangp/teaching/courses/RE2009Fall/REWiki/

[11] L. Abeti, P. Ciancarini, and R. Moretti. "Wiki-based Requirements Management for Business Process Reengineering". In Proceedings of the 4th International Symposium on Wikis(WikiSym), pages 45-50. ACM, 2008.

[12] S. Lohmann, P. Heim, S. Auer, S. Dietzold, and T. Riechert. "Semantifying requirementsengineering - the softwiki approach". In Proceedings of the 4th International ConferenceonSemantic Technologies (I-SEMANTICS), pages 182-185. ACM, 2008. 